Research Article

\title{
Preview Tracking Control for Continuous-Time Singular Interconnected Systems
}

\author{
Hao Xie, ${ }^{1}$ Fucheng Liao $\mathbb{D}^{1}{ }^{1}$ and Jiamei Deng ${ }^{2}$ \\ ${ }^{1}$ School of Mathematics and Physics, University of Science and Technology Beijing, Beijing 100083, China \\ ${ }^{2}$ School of Built Environment, Engineering and Computing, Leeds Beckett University, Leeds LS6 3QS, UK \\ Correspondence should be addressed to Fucheng Liao; fcliao@ustb.edu.cn
}

Received 28 September 2019; Accepted 26 November 2019; Published 19 December 2019

Academic Editor: Hung-Yuan Chung

Copyright $\odot 2019$ Hao Xie et al. This is an open access article distributed under the Creative Commons Attribution License, which permits unrestricted use, distribution, and reproduction in any medium, provided the original work is properly cited.

\begin{abstract}
This paper proposes and investigates a problem of preview tracking control for a class of continuous-time singular interconnected systems. Firstly, the related items are deleted to obtain several isolated subsystems with low dimensions. An error system is constructed for each isolated subsystem so that the tracking error is included in the state vector of the error system; then, the tracking problem is transformed into a regulation problem. Secondly, the preview tracking controller is designed for each error system and obtained controllers are combined as the controller of the error system of the singular interconnected system. Thirdly, the Lyapunov function method is utilized to determine the constraints of the related terms so that the closed-loop system of the error system of the singular interconnected system is stable under the action of the controller obtained. Finally, the preview tracking controller of the singular interconnected system is obtained from the relationship between the error system and the original system. A numerical simulation algorithm for continuous-time singular systems is also proposed in this paper. The numerical simulation illustrates the effectiveness of the theoretical results.
\end{abstract}

\section{Introduction}

Compared with the normal system, the singular system is a kind of dynamic system with a more extensive form and wide application background. Singular systems exist in many fields, such as economic management, bioengineering systems, aerospace technology, and robotic systems [1-3]. From the early 1970s to the end of the 1980s, fruitful achievements have been made in the research of minimum realization problem, observer design, and stability of singular systems [4-6]. Since the 1990s, research on singular systems has been developed from basis to depth, covering various topics from linear to nonlinear, from time-invariant to time-varying systems, and from linear quadratic optimal control to $H_{\infty}$ control [7-9]. Because the singular system model comes from the engineering practice, the research of the theory of singular systems must serve the practical application finally. In many practical engineering situations, there are usually large-scale, complex structures, many factors, and functional comprehensive of the systems, that is, large-scale systems, also known as interconnected systems, which leads to the study of singular interconnected systems. For singular interconnected systems, if the controller is designed using a centralized control scheme, it will be difficult to deal with too much information, which makes the design difficult to achieve. Therefore, a decentralized-aggregation method is adopted to design the controller $[10,11]$. This is the decomposition method of large-scale systems. Firstly, the related terms are deleted artificially, and several lower-dimensional systems (also called isolated subsystems) are obtained. The controllers satisfying the requirements are designed, respectively. Then, the controller of the interconnected system is obtained through a certain method of synthesis $[12,13]$. In recent years, there exist many research studies on control theory of singular interconnected systems and many significant results have been obtained [14, 15]. Wo et al. studied robust stabilization of discrete-time singular interconnected systems with parameter uncertainties [11]. Lu and Ho gave sufficient conditions for stability and decentralized stabilization of a class of 
singular interconnected systems utilizing linear matrix inequalities (LMIs) [16]. Employing the singular Lyapunov matrix equation, system decomposition method, and matrix theory, Chen and Ding probed the stabilization problem of singular linear interconnected systems with output feedback and provided sufficient conditions for asymptotic stability and instability of corresponding closed-loop singular interconnected systems [17].

The future values of desired tracking signals or disturbance signals of some practical control systems are partly or completely known, such as flight routes of aircraft, processing paths of numerically controlled machine tools, and driving paths of vehicles. Utilizing the known future information to improve the quality of the closed-loop system is known as the preview control problem. At present, the research on optimal preview control of linear quadratic form is in-depth. By adopting the method of augmented systems, Katayama and Hirono discussed the optimal preview control problem for continuous-time [18]. On the basis of [18], Liao et al. studied the situation where both the desired tracking signal and the disturbance signal are previewable at the same time [19]. In recent years, the theory of preview control for stochastic systems, the theory of robust preview control, and the theory of preview control for multirate systems have been developed [20-22]. $\mathrm{Wu}$ et al. investigate the optimal preview control problem for a class of continuous-time stochastic systems by constructing an auxiliary system [20]. Li and Liao combine parameter-dependent Lyapunov stability theory with function method and LMI techniques to solve the static output feedback preview tracking control problem for a class of polyhedral uncertain discrete-time systems [21]. At the same time, preview control has been successfully used in many practical applications, such as vehicle active suspension systems, electromechanical servo systems, robots and aircraft [23-25].

On the premise that both the theory of singular interconnected systems and preview control have made considerable progress, it is of great theoretical and practical significance to combine them. So far, there is no literature on preview control of singular interconnected systems. In view of this, this paper proposes and studies the preview tracking control problem for continuous-time singular interconnected systems.

Research contents are arranged as follows: Section 2 presents a class of preview tracking control problems for continuous-time singular interconnected systems and gives necessary assumptions. The designs of the error system controller of isolated subsystems and the controller of the singular interconnected system are discussed in Sections 3 and 4 , respectively. Section 5 is numerical simulation. The method presented is not only applicable to this paper but also to general continuous-time singular systems. Finally, a brief conclusion is given in Section 6 .

Throughout this paper, $A \in R^{n \times m}$ represents $A$ as the real matrix of $n \times m ; Q>0 \quad(Q \geq 0)$ shows the matrix $Q$ symmetric positive definite (semi-positive definite); $\operatorname{deg}(\operatorname{det}(\cdot))$ is the degree of the determinant; and $\|\cdot\|$ represents the matrix norm derived from the Euclidean norm of a vector.

\section{Problem Formulation and Basic Assumptions}

Consider a continuous-time singular interconnected system as follows:

$$
\left\{\begin{array}{l}
E_{i} \dot{x}_{i}(t)=A_{i} x_{i}(t)+\sum_{\substack{j=1 \\
j \neq i}}^{N} A_{i j} x_{j}(t)+B_{i} u_{i}(t), \quad i=1,2, \ldots, N, \\
y(t)=\sum_{i=1}^{N} y_{i}(t)=\sum_{i=1}^{N} C_{i} x_{i}(t),
\end{array}\right.
$$

where $x_{i}(t) \in R^{n_{i}}, \quad u_{i}(t) \in R^{m_{i}}, \quad y_{i}(t), y(t) \in R^{p}, \quad E_{i}, A_{i} \in$ $R^{n_{i} \times n_{i}}, A_{i j} \in R^{n_{i} \times n_{j}}, B_{i} \in R^{n_{i} \times m_{i}}$, and $C_{i} \in R^{p \times n_{i}}$ are constant matrices; $A_{i j}(i \neq j)$ is the related matrix; and $\operatorname{rank}\left(E_{i}\right)=$ $q_{i}<n_{i}(i=1,2, \ldots, N)$.

Let $y_{d}(t) \in R^{p}$ be the desired tracking signal or the reference signal.

Firstly, some necessary assumptions are given as follows:

A1: assume that $\left(E_{i}, A_{i}\right)$ is regular, that is to say, there exists $s_{i} \in C$, so that $\operatorname{det}\left(s_{i} E_{i}-A_{i}\right) \neq 0(i=1,2, \ldots, N)$ [26]

A2: assume that $\left(E_{i}, A_{i}\right)$ is impulse free, that is to say, for any $s \in C, \operatorname{deg}\left(\operatorname{det}\left(s E_{i}-A_{i}\right)\right)=\operatorname{rank}\left(E_{i}\right)$ holds $(i=1,2, \ldots, N)[26]$

A3: assume that $\left(E_{i}, A_{i}, B_{i}\right)$ is stabilizable, which means $\operatorname{rank}\left[s E_{i}-A_{i} B_{i}\right]=n_{i}$ holds for any complex $s$ satisfying $\operatorname{Re}(s) \geq 0(i=1,2, \ldots, N)[26]$

A4: assume that the matrix $\left[\begin{array}{cc}A_{i} & B_{i} \\ C_{i} & 0\end{array}\right]$ is of full row rank
$(i=1,2, \ldots, N)$

A5: assume that $\left(E_{i}, A_{i}, C_{i}\right)$ is detectable, which means $\operatorname{rank}\left[\begin{array}{c}s E_{i}-A_{i} \\ C_{i}\end{array}\right]=n_{i}$ holds for any complex $s$ satisfying $\operatorname{Re}(s) \geq 0(i=1,2, \ldots, N)[26]$

A6: assume that desired tracking signal $y_{d}(t)$ is a piecewise continuously differentiable function satisfying

$$
\begin{aligned}
& \lim _{t \longrightarrow \infty} y_{d}(t)=\bar{y}_{d}, \\
& \lim _{t \longrightarrow \infty} \dot{y}_{d}(t)=0,
\end{aligned}
$$

where $\bar{y}_{d}$ is a constant vector. Besides, $y_{d}(t)$ is previewable; in other words, at the time $t, y_{d}(\tau)\left(t \leq \tau \leq t+l_{r}\right)$ is available, where $l_{r}$ is called the preview length.

The system

$$
\left\{\begin{array}{l}
E_{i} \dot{x}_{i}(t)=A_{i} x_{i}(t)+B_{i} u_{i}(t), \\
y_{i}(t)=C_{i} x_{i}(t)
\end{array}\right.
$$

is called an isolated subsystem of (1). There are $N$ isolated subsystems $(i=1,2, \ldots, N)$.

Remark 1. The establishment of A1 and A2 indicates that the $N$ isolated subsystems of System (1) are regular and 
impulse free. The establishment of A3 and A5 indicates that the $N$ isolated subsystems of System (1) are stabilizable and detectable. A6 is the standard assumption of preview control.

The tracking error of System (1) is defined as follows:

$$
e(t)=y(t)-y_{d}(t)
$$

The objective of this paper is to design a controller with preview action so that $y(t)$ is able to track the desired tracking signal $y_{d}(t)$ without static error; in other words,

$$
\lim _{t \longrightarrow \infty} e(t)=\lim _{t \longrightarrow \infty}\left[y(t)-y_{d}(t)\right]=0 .
$$

\section{Controller of the Error System of Isolated Subsystems}

For the sake of designing the controller, decentralized control is adopted. Firstly, the controller is designed for each isolated subsystem of System (1). Then, all the controllers are combined as the controllers of singular interconnected systems, and the Lyapunov function method is utilized to give the restriction conditions of the related terms, such that the output of closed-loop systems of singular interconnected systems is able to track $y_{d}(t)$.

According to the form of System (1) output equation, the tracking error $e(t)$ is rewritten as

$$
e(t)=\sum_{i=1}^{N} e_{i}(t)=\sum_{i=1}^{N}\left[y_{i}(t)-\alpha_{i} y_{d}(t)\right]
$$

where $\alpha_{i}(i=1,2, \ldots, N)$ are constants which satisfy $\sum_{i=1}^{N} \alpha_{i}=1$. It is known from (6) that if for any $e_{i}(t)=y_{i}(t)-$ $\alpha_{i} y_{d}(t) \quad(i=1,2, \ldots, N)$, there is $\lim _{t \longrightarrow \infty} e_{i}(t)=0$, then $\lim _{t \rightarrow \infty} e(t)=0$.

Remark 2. $y_{i}(t)$ can be understood as the output of $i$-th subsystem. (6) means that if output $y_{i}(t)$ of $i$-th subsystem tracks $\alpha_{i} y_{d}(t) \quad(i=1,2, \ldots, N)$, then the output $y(t)=\sum_{i=1}^{N} y_{i}(t)$ of System (1) can track $y_{d}(t)$. The parameter $\alpha_{i}(i=1,2, \ldots, N)$ gives us the freedom of choice. For example, let $\alpha_{1}=\alpha_{2}=\cdots=\alpha_{N}=1 / N$, which means that all $y_{i}(t)$ keep track of $(1 / N) y_{d}(t)$. If $\alpha_{i}=0$, this indicates that the output of the $i$-th subsystem tracks the zero vector, then the task of tracking $y_{d}(t)$ is completed by the output of other subsystems cooperatively, etc.

The optimal control technique is employed to design the controller of System (3). For a given $i(i=1,2, \ldots, N)$, the quadratic performance index function is introduced as follows:

$$
J_{i}=\frac{1}{2} \int_{0}^{\infty}\left[e_{i}^{T}(t) Q_{e_{i}} e_{i}(t)+\dot{u}_{i}^{T}(t) R_{i} \dot{u}_{i}(t)\right] \mathrm{d} t,
$$

where $Q_{e_{i}} \in R^{p \times p}$ and $R_{i} \in R^{m_{i} \times m_{i}}$ are positive definite matrices. It has been pointed out in [19] that the introduction of $\dot{u}_{i}(t)$ in the performance index function can make the controller contain integrators, which helps to eliminate the static output errors in the closed-loop system.
An error system is constructed for the isolated subsystem by utilizing the usual preview control method, so that the tracking error becomes a part of the state vector of the error system. As a result, the tracking problem of the isolated subsystem is turned into a regulation problem of the error system. Since the error system of the singular interconnected system is needed later in this paper, for the purpose of avoiding repetition, the error system of System (1) is firstly constructed, then the related term is removed to obtain the error system of isolated subsystems.

Differentiate both sides of the state equation of System (1) to obtain

$$
E_{i} \ddot{x}_{i}(t)=A_{i} \dot{x}_{i}(t)+\sum_{\substack{j=1 \\ j \neq i}}^{N} A_{i j} \dot{x}_{j}(t)+B_{i} \dot{u}_{i}(t), \quad i=1,2, \ldots, N .
$$

Then, differentiate $e_{i}(t)=y_{i}(t)-\alpha_{i} y_{d}(t) \quad(i=1$, $2, \ldots, N)$ to obtain

$$
\dot{e}_{i}(t)=\dot{y}_{i}(t)-\alpha_{i} \dot{y}_{d}(t)=C_{i} \dot{x}_{i}(t)-\alpha_{i} \dot{y}_{d}(t), \quad i=1,2, \ldots, N .
$$

Combining (8) and (9), we have

$$
\begin{array}{r}
\bar{E}_{i} \dot{X}_{i}(t)=\bar{A}_{i} X_{i}(t)+\sum_{\substack{j=1 \\
j \neq i}}^{N} \bar{A}_{i j} X_{j}(t)+\bar{B}_{i} \dot{u}_{i}(t)-G_{i} \dot{y}_{d}(t), \\
i=1,2, \ldots, N,
\end{array}
$$

where

$$
\begin{aligned}
X_{i}(t) & =\left[\begin{array}{c}
e_{i}(t) \\
\dot{x}_{i}(t)
\end{array}\right] \in R^{p+n_{i}}, \\
\bar{E}_{i} & =\left[\begin{array}{cc}
I_{p} & 0 \\
0 & E_{i}
\end{array}\right] \in R^{\left(p+n_{i}\right) \times\left(p+n_{i}\right)}, \\
\bar{A}_{i} & =\left[\begin{array}{cc}
0 & C_{i} \\
0 & A_{i}
\end{array}\right] \in R^{\left(p+n_{i}\right) \times\left(p+n_{i}\right)}, \\
\bar{A}_{i j} & =\left[\begin{array}{cc}
0 & 0 \\
0 & A_{i j}
\end{array}\right] \in R^{\left(p+n_{i}\right) \times\left(p+n_{j}\right)}, \\
\bar{B}_{i} & =\left[\begin{array}{c}
0 \\
B_{i}
\end{array}\right] \in R^{\left(p+n_{i}\right) \times m_{i}}, \\
G_{i} & =\left[\begin{array}{c}
\alpha_{i} I_{p} \\
0
\end{array}\right] \in R^{\left(p+n_{i}\right) \times p} .
\end{aligned}
$$

System (10) is the error system of singular interconnected System (1).

The error system of the isolated subsystem can be obtained by taking all the associated matrices $\bar{A}_{i j}(i \neq j)$ in (10) as zero matrices. The error system of the $i$-th isolated subsystem becomes 


$$
\bar{E}_{i} \dot{X}_{i}(t)=\bar{A}_{i} X_{i}(t)+\bar{B}_{i} \dot{u}_{i}(t)-G_{i} \dot{y}_{d}(t) .
$$

Adopting the state vector of formula (12), the performance index function (7) can be written as

$$
J_{i}=\frac{1}{2} \int_{0}^{\infty}\left[X_{i}^{T}(t) \bar{Q}_{i} X_{i}(t)+\dot{u}_{i}^{T}(t) R_{i} \dot{u}_{i}(t)\right] \mathrm{d} t,
$$

where $\bar{Q}_{i}=\left[\begin{array}{cc}Q_{e_{i}} & 0 \\ 0 & 0\end{array}\right] \in R^{\left(p+n_{i}\right) \times\left(p+n_{i}\right)}$.

For the sake of making full use of the mature controller design methods and results in the optimal preview control theory of normal systems, System (12) needs to be changed into a normal system and an algebraic equation by restricted equivalent transformation [26].

Notice that if $\mathrm{A} 1$ and $\mathrm{A} 2$ are true, then $\left(\bar{E}_{i}, \bar{A}_{i}\right)$ is regular and impulse free. This is proved as follows.

In the light of [26], $\left(E_{i}, A_{i}\right)$ is regular and impulse free if and only if there are nonsingular matrices $S_{i}$ and $T_{i}$, such that

$$
\begin{aligned}
S_{i} E_{i} T_{i} & =\left[\begin{array}{cc}
I_{q_{i}} & 0 \\
0 & 0
\end{array}\right], \\
S_{i} A_{i} T_{i} & =\left[\begin{array}{cc}
A_{i 1} & 0 \\
0 & I_{n_{i}-q_{i}}
\end{array}\right] .
\end{aligned}
$$

Taking the nonsingular matrix $S_{i i}=\left[\begin{array}{cc}I_{p} & 0 \\ 0 & S_{i}\end{array}\right]$ and $T_{i i}=\left[\begin{array}{cc}I_{p} & 0 \\ 0 & T_{i}\end{array}\right]$, there are

$$
\begin{gathered}
S_{i i} \bar{E}_{i} T_{i i}=\left[\begin{array}{cc}
I_{p} & 0 \\
0 & S_{i} E_{i} T_{i}
\end{array}\right]=\left[\begin{array}{ccc}
I_{p} & 0 & 0 \\
0 & I_{q_{i}} & 0 \\
0 & 0 & 0
\end{array}\right], \\
S_{i i} \bar{A}_{i} T_{i i}=\left[\begin{array}{cc}
0 & C_{i} T_{i} \\
0 & S_{i} A_{i} T_{i}
\end{array}\right]=\left[\begin{array}{ccc}
0 & \widetilde{C}_{i 1} & \widetilde{C}_{i 2} \\
0 & A_{i 1} & 0 \\
0 & 0 & I_{n_{i}-q_{i}}
\end{array}\right] . \\
\text { Let } \bar{S}_{i}=\left[\begin{array}{ccc}
I_{p} & 0 & -\widetilde{C}_{i 2} \\
0 & I_{q_{i}} & 0 \\
0 & 0 & I_{n_{i}-q_{i}}
\end{array}\right] S_{i i} \text { and } \bar{T}_{i}=T_{i i} \text {. Obviously, } \bar{S}_{i}
\end{gathered}
$$
and $\bar{T}_{i}$ are nonsingular and there are

$$
\begin{aligned}
& \bar{S}_{i} \bar{E}_{i} \bar{T}_{i}=\left[\begin{array}{ccc}
I_{p} & 0 & -\widetilde{C}_{i 2} \\
0 & I_{q_{i}} & 0 \\
0 & 0 & I_{n_{i}-q_{i}}
\end{array}\right]\left[\begin{array}{ccc}
I_{p} & 0 & 0 \\
0 & I_{q_{i}} & 0 \\
0 & 0 & 0
\end{array}\right]=\left[\begin{array}{ccc}
I_{p} & 0 & 0 \\
0 & I_{q_{i}} & 0 \\
0 & 0 & 0
\end{array}\right], \\
& \bar{S}_{i} \bar{A}_{i} \bar{T}_{i}=\left[\begin{array}{ccc}
I_{p} & 0 & -\widetilde{C}_{i 2} \\
0 & I_{q_{i}} & 0 \\
0 & 0 & I_{n_{i}-q_{i}}
\end{array}\right]\left[\begin{array}{ccc}
0 & \widetilde{C}_{i 1} & \widetilde{C}_{i 2} \\
0 & A_{i 1} & 0 \\
0 & 0 & I_{n_{i}-q_{i}}
\end{array}\right]=\left[\begin{array}{ccc}
0 & \widetilde{C}_{i 1} & 0 \\
0 & A_{i 1} & 0 \\
0 & 0 & I_{n_{i}-q_{i}}
\end{array}\right] .
\end{aligned}
$$

This means that $\left(\bar{E}_{i}, \bar{A}_{i}\right)$ is regular and impulse free [26]. In addition, the right side of the two formulas is denoted as $\widehat{E}_{i}$ and $\widehat{A}_{i}$, respectively, i.e., $\widehat{E}_{i}=\bar{S}_{i} \bar{E}_{i} \bar{T}_{i}=\left[\begin{array}{cc}I_{p+q_{i}} & 0 \\ 0 & 0\end{array}\right]$ and $\widehat{A}_{i}=\bar{S}_{i} \bar{A}_{i} \bar{T}_{i}=\left[\begin{array}{cc}\widetilde{A}_{i 1} & 0 \\ 0 & I_{n_{i}-q_{i}}\end{array}\right]$. For System (12), introducing a nonsingular linear transformation $X_{i}(t)=\bar{T}_{i} \bar{X}_{i}(t)$ and then premultiplying a nonsingular matrix $\bar{S}_{i}$ on both sides, we obtain

$$
\widehat{E}_{i} \dot{\bar{X}}_{i}(t)=\widehat{A}_{i} \bar{X}_{i}(t)+\widehat{B}_{i} \dot{u}_{i}(t)-\widehat{G}_{i} \dot{y}_{d}(t),
$$

where

$$
\begin{aligned}
\widehat{B}_{i} & =\bar{S}_{i} \bar{B}_{i}, \\
\widehat{G}_{i} & =\bar{S}_{i} G_{i} .
\end{aligned}
$$

Denote

$$
\bar{X}_{i}(t)=\left[\begin{array}{l}
\bar{X}_{i 1}(t) \\
\bar{X}_{i 2}(t)
\end{array}\right]
$$

where $\bar{X}_{i 1}(t) \in R^{p+q_{i}}$ and $\bar{X}_{i 2}(t) \in R^{n_{i}-q_{i}}$. Blocks $\widehat{B}_{i}$ and $\widehat{G}_{i}$ are as follows:

$$
\begin{aligned}
& \widehat{B}_{i}=\left[\begin{array}{l}
\widetilde{B}_{i 1} \\
\widetilde{B}_{i 2}
\end{array}\right], \\
& \widehat{G}_{i}=\left[\begin{array}{c}
\widetilde{G}_{i 1} \\
\widetilde{G}_{i 2}
\end{array}\right] .
\end{aligned}
$$

Then, (17) can be written as

$$
\begin{aligned}
\dot{\bar{X}}_{i 1}(t) & =\widetilde{A}_{i 1} \bar{X}_{i 1}(t)+\widetilde{B}_{i 1} \dot{u}_{i}(t)-\widetilde{G}_{i 1} \dot{y}_{d}(t), \\
0 & =\bar{X}_{i 2}(t)+\widetilde{B}_{i 2} \dot{u}_{i}(t)-\widetilde{G}_{i 2} \dot{y}_{d}(t) .
\end{aligned}
$$

Using the state vector of (17) (or (21)), the performance index function (13) can be written as

$$
J_{i}=\frac{1}{2} \int_{0}^{\infty}\left[\bar{X}_{i}^{T}(t) \widehat{Q}_{i} \bar{X}_{i}(t)+\dot{u}_{i}^{T}(t) R_{i} \dot{u}_{i}(t)\right] \mathrm{d} t,
$$

where $\widehat{Q}_{i}=\bar{T}_{i}^{T} \bar{Q}_{i} \bar{T}_{i} \in R^{\left(p+n_{i}\right) \times\left(p+n_{i}\right)}$.

Note that the restricted equivalence transformation does not change the dynamic characteristics of singular systems, such as regularity, impulse free, stability, stabilization, and detectability [26]. Therefore, System (12) can be studied through System (17) (or (21)). That is, only the controller of System (21a) needs to be designed.

By utilizing the state vector of formula (21a), the performance index function (22) can be written as

$$
J_{i}=\frac{1}{2} \int_{0}^{\infty}\left[\bar{X}_{i 1}^{T}(t) \widetilde{Q}_{i} \bar{X}_{i 1}(t)+\dot{u}_{i}^{T}(t) R_{i} \dot{u}_{i}(t)\right] \mathrm{d} t,
$$

where $\widetilde{Q}_{i}=\left[\begin{array}{cc}Q_{e_{i}} & 0 \\ 0 & 0\end{array}\right] \in R^{\left(p+q_{i}\right) \times\left(p+q_{i}\right)}$.

From the known conclusion [19], Theorem 1 can be proved directly.

Theorem 1. If $\left(\widetilde{A}_{i 1}, \widetilde{B}_{i 1}\right)$ is stabilizable, $\left(\widetilde{Q}_{i}^{1 / 2}, \widetilde{A}_{i 1}\right)$ is detectable and A6 holds; then, the controller of System (21a), which minimizes the performance index function (14), has the form of

$$
\dot{u}_{i}(t)=-R_{i}^{-1} \widetilde{B}_{i 1}^{T} P_{i} \bar{X}_{i 1}(t)+g_{i}(t),
$$


where

$$
g_{i}(t)=R_{i}^{-1} \widetilde{B}_{i 1}^{T} \int_{0}^{l_{r}} \exp \left(\sigma \widetilde{A}_{c i}^{T}\right) P_{i} \widetilde{G}_{i 1} \dot{y}_{d}(t+\sigma) \mathrm{d} \sigma .
$$

$\widetilde{A}_{c i}$ is a stable matrix and its expression is

$$
\widetilde{A}_{c i}=\widetilde{A}_{i 1}-\widetilde{B}_{i 1} R_{i}^{-1} \widetilde{B}_{i 1}^{T} P_{i} .
$$

$P_{i}$ is the positive definite solution of the following Riccati equation:

$$
\widetilde{A}_{i 1}^{T} P_{i}+P_{i} \widetilde{A}_{i 1}-P_{i} \widetilde{B}_{i 1} R_{i}^{-1} \widetilde{B}_{i 1}^{T} P_{i}+\widetilde{Q}_{i}=0 .
$$

Next, the optimal preview controller for System (12) and the performance index (13) is given. Firstly, the following two lemmas are established.

Lemma 1 (see [26]). For a given $i(i=1,2, \ldots, N),\left(\widetilde{A}_{i 1}, \widetilde{B}_{i 1}\right)$ is stabilizable if and only if $\left(\bar{E}_{i}, \bar{A}_{i}, \bar{B}_{i}\right)$ is stabilizable.

The proof of Lemma 1 is shown in Theorem 8.4.1 in literature [26].

Remark 3. Lemma 1 shows that the stabilization of singular System (12) is determined only by normal System (21a) obtained through its restricted equivalent transformation.

Lemma 2. For a given $i(i=1,2, \ldots, N),\left(\widetilde{Q}_{i}^{1 / 2}, \widetilde{A}_{i 1}\right)$ is detectable if and only if $\left(\bar{E}_{i}, \bar{A}_{i}, \bar{Q}_{i}{ }^{1 / 2}\right)$ is detectable.

Proof. This lemma can be obtained according to Theorem 8.3.2 in literature [26] and by the method of proving Theorem 8.4.1 in literature [26]. It is omitted here.

Under the performance index function (13), the optimal preview controller of System (12) is given by Theorem 2 .

Theorem 2. If $\left(E_{i}, A_{i}\right)$ is regular and impulse free, $\left(\bar{E}_{i}, \bar{A}_{i}, \bar{B}_{i}\right)$ is stabilizable, $\left(\bar{E}_{i}, \bar{A}_{i}, \bar{Q}_{i}^{1 / 2}\right)$ is detectable, and $A 6$ holds; then under the performance index function (13), the optimal preview controller of System (12) is

$$
\dot{u}_{i}(t)=-R_{i}^{-1} \widetilde{B}_{i 1}^{T} P_{i} U_{i} X_{i}(t)+g_{i}(t)
$$

where $U_{i}=\left[\begin{array}{ll}I_{p+q_{i}} & 0\end{array}\right] \bar{T}_{i}^{-1}$. Besides, $\left(\bar{E}_{i}, \bar{A}_{i}-\bar{B}_{i} R_{i}^{-1} \widetilde{B}_{i 1}^{T} P_{i} U_{i}\right)$ is admissible.

The so-called admissibility refers to regularity, impulse free, and stabilization [26]. Therefore, $\left(\bar{E}_{i}, \bar{A}_{i}-\bar{B}_{i} R_{i}^{-1} \widetilde{B}_{i 1}^{T} P_{i} U_{i}\right)$ is admissible which means that $\left(\bar{E}_{i}, \bar{A}_{i}-\bar{B}_{i} R_{i}^{-1} \widetilde{B}_{i 1}^{T} P_{i} U_{i}\right)$ is regular, impulse free, and stable.

Proof. When $\left(E_{i}, A_{i}\right)$ is regular and impulse free, the restricted equivalent transformation of the above system (13) holds. In addition, according to Lemma 1 and Lemma 2, the condition of Theorem 2 can guarantee that Theorem 1 is true. Therefore, the controller (24) is obtained according to Theorem 1. Because $\bar{T}_{i}$ is an invertible matrix in the transformation $X_{i}(t)=\bar{T}_{i} \bar{X}_{i}(t)$, the optimal preview controller (24) for System (21a) and the performance index (23) is the optimal preview controller for System (12) under the performance index function (13).

Thanks to $X_{i}(t)=\bar{T}_{i} \bar{X}_{i}(t)$ and $\bar{X}_{i}(t)=\left[\begin{array}{l}\bar{X}_{i 1}(t) \\ \bar{X}_{i 2}(t)\end{array}\right]$, there is $\left[\begin{array}{l}\bar{X}_{i 1}(t) \\ \bar{X}_{i 2}(t)\end{array}\right]=\bar{T}_{i}^{-1} X_{i}(t)$. Therefore, $\bar{X}_{i 1}(t)=\left[\begin{array}{ll}I_{p+q_{i}} & 0\end{array}\right]\left[\begin{array}{l}\bar{X}_{i 1}(t) \\ \bar{X}_{i 2}(t)\end{array}\right]=\left[\begin{array}{ll}I_{p+q_{i}} & 0\end{array}\right] \bar{T}_{i}^{-1} X_{i}(t)=U_{i} X_{i}(t)$.

Substitute (29) into (24) to get (28).

The following proves that $\left(\bar{E}_{i}, \bar{A}_{i}-\bar{B}_{i} R_{i}^{-1} \widetilde{B}_{i 1}^{T} P_{i} U_{i}\right)$ is admissible.

$\left.\begin{array}{l}\text { Taking } \widetilde{S}_{i}=\bar{S}_{i} \text { and } \widetilde{T}_{i}=\bar{T}_{i}\left[\begin{array}{cc}I_{p+q_{i}} & 0 \\ \text { not singular. Because }\end{array}\right], \widetilde{S}_{i 2} R_{i}^{-1} \widetilde{B}_{i 1}^{T} P_{i} \\ I_{n_{i}-q_{i}}\end{array}\right] \widetilde{T}_{i}$

$$
\begin{aligned}
\widetilde{S}_{i} \bar{E}_{i} \widetilde{T}_{i} & =\bar{S}_{i} \bar{E}_{i} \bar{T}_{i}\left[\begin{array}{cc}
I_{p+q_{i}} & 0 \\
\widetilde{B}_{i 2} R_{i}^{-1} \widetilde{B}_{i 1}^{T} P_{i} & I_{n_{i}-q_{i}}
\end{array}\right]=\left[\begin{array}{cc}
I_{p_{i}+q_{i}} & 0 \\
0 & 0
\end{array}\right]\left[\begin{array}{cc}
I_{p+q_{i}} & 0 \\
\widetilde{B}_{i 2} R_{i}^{-1} \widetilde{B}_{i 1}^{T} P_{i} & I_{n_{i}-q_{i}}
\end{array}\right]=\left[\begin{array}{cc}
I_{p_{i}+q_{i}} & 0 \\
0 & 0
\end{array}\right], \\
\widetilde{S}_{i}\left(\bar{A}_{i}-\bar{B}_{i} R_{i}^{-1} \widetilde{B}_{i 1}^{T} P_{i} U_{i}\right) \widetilde{T}_{i} & =\left(\bar{S}_{i} \bar{A}_{i} \bar{T}_{i}-\bar{S}_{i} \bar{B}_{i} R_{i}^{-1} \widetilde{B}_{i 1}^{T} P_{i} U_{i} \bar{T}_{i}\right)\left[\begin{array}{cc}
I_{p+q_{i}} & 0 \\
\widetilde{B}_{i 2} R_{i}^{-1} \widetilde{B}_{i 1}^{T} P_{i} & I_{n_{i}-q_{i}}
\end{array}\right] \\
& =\left(\left[\begin{array}{cc}
\widetilde{A}_{i 1} & 0 \\
0 & I_{n_{i}-q_{i}}
\end{array}\right]-\left[\begin{array}{c}
\widetilde{B}_{i 1} \\
\widetilde{B}_{i 2}
\end{array}\right] R_{i}^{-1} \widetilde{B}_{i 1}^{T} P_{i}\left[\begin{array}{ll}
I_{p+q_{i}} & 0
\end{array}\right]\right)\left[\begin{array}{cc}
I_{p+q_{i}} & 0 \\
\widetilde{B}_{i 2} R_{i}^{-1} \widetilde{B}_{i 1}^{T} P_{i} & I_{n_{i}-q_{i}}
\end{array}\right] \\
& =\left[\begin{array}{cc}
\widetilde{A}_{i 1}-\widetilde{B}_{i 1} R_{i}^{-1} \widetilde{B}_{i 1}^{T} P_{i} & 0 \\
-\widetilde{B}_{i 2} R_{i}^{-1} \widetilde{B}_{i 1}^{T} P & I_{n_{i}-q_{i}}
\end{array}\right]\left[\begin{array}{cc}
I_{p+q_{i}} & 0 \\
\widetilde{B}_{i 2} R_{i}^{-1} \widetilde{B}_{i 1}^{T} P_{i} & I_{n_{i}-q_{i}}
\end{array}\right] \\
& =\left[\begin{array}{cc}
\widetilde{A}_{i 1}-\widetilde{B}_{i 1} R_{i}^{-1} \widetilde{B}_{i 1}^{T} P_{i} & 0 \\
0 & I_{n_{i}-q_{i}}
\end{array}\right],
\end{aligned}
$$

there is 


$$
\begin{aligned}
& \widetilde{S}_{i}\left[\begin{array}{ll}
\bar{E}_{i} & \bar{A}_{i}-\bar{B}_{i} R_{i}^{-1} \widetilde{B}_{i 1}^{T} P_{i}
\end{array}\right] \widetilde{T}_{i} \\
& =\left[\begin{array}{cccc}
I_{p_{i}+q_{i}} & 0 & \widetilde{A}_{i 1}-\widetilde{B}_{i 1} R_{i}^{-1} \widetilde{B}_{i 1}^{T} P_{i} & 0 \\
0 & 0 & 0 & I_{n_{i}-q_{i}}
\end{array}\right] .
\end{aligned}
$$

This indicates that $\left(\bar{E}_{i}, \bar{A}_{i}-\bar{B}_{i} R_{i}^{-1} \widetilde{B}_{i 1}^{T} P_{i} U_{i}\right)$ is regular and impulse free [26]. Notice that $\widetilde{A}_{i 1}-\widetilde{B}_{i 1} R_{i}^{-1} \widetilde{B}_{i 1}^{T} P_{i}$ is stable, which means that $\left(\bar{E}_{i}, \bar{A}_{i}-\bar{B}_{i} R_{i}^{-1} \widetilde{B}_{i 1}^{T} P_{i} U_{i}\right)$ is also stable [26]. Thus, Theorem 2 is proved.

\section{Design of a Preview Controller for Singular Interconnected Systems}

$\dot{u}_{i}(i=1,2, \ldots, N)$ given by equation (28) are employed to construct a vector

$$
\dot{u}(t)=\left[\begin{array}{llll}
\dot{u}_{1}^{T}(t) & \dot{u}_{2}^{T}(t) & \cdots & \dot{u}_{N}^{T}(t)
\end{array}\right]^{T},
$$

as the controller of error System (10). Next, determine the conditions in which the related term should satisfy so that the state vector $X_{i}(t)(i=1,2, \ldots, N)$ of the closed-loop system of System (10) is asymptotically stable to zero vector. Firstly, the following lemma is given.

Lemma 3 (see [27]). For singular systems, the following propositions are equivalent:

(i) $E \dot{x}(t)=A x(t)$ or $(E, A)$ is admissible

(ii) For any given positive definite matrix $W$, there exists a matrix $\bar{P}$ so that

$$
\left\{\begin{array}{l}
\bar{P}^{T} A+A^{T} \bar{P}=-W, \\
E^{T} \bar{P}=\bar{P}^{T} E \geq 0,
\end{array}\right.
$$

holds

(iii) The singular Lyapunov function $V(E x)=x^{T} E^{T} \bar{P} x$ satisfies

$$
\frac{\mathrm{d} V(E x(t))}{\mathrm{d} t}<0
$$

where $x(t) \neq 0, E^{T} \bar{P}=\bar{P}^{T} E \geq 0$, and $\operatorname{rank}\left(E^{T} \bar{P}\right)=\operatorname{rank}(E)$

Substitute (32) into System (10) to get the closed-loop system:

$$
\begin{gathered}
\bar{E}_{i} \dot{X}_{i}(t)=\left(\bar{A}_{i}-\bar{B}_{i} R_{i}^{-1} \widetilde{B}_{i 1}^{T} P_{i} U_{i}\right) X_{i}(t)+\sum_{\substack{j=1 \\
j \neq i}}^{N} \bar{A}_{i j} X_{j}(t)+\eta_{i}(t), \\
i=1,2, \ldots, N,
\end{gathered}
$$

where

$$
\eta_{i}(t)=\bar{B}_{i} g_{i}(t)-G_{i} \dot{y}_{d}(t), \quad i=1,2, \ldots, N .
$$

Theorem 3 can be obtained, which gives the condition that the state vector of System (35) is asymptotically stable to the zero vector.

Theorem 3. If

(I) $\left(E_{i}, A_{i}\right)$ is regular and impulse free, $\left(\bar{E}_{i}, \bar{A}_{i}, \bar{B}_{i}\right)$ is stabilizable, and $\left(\bar{E}_{i}, \bar{A}_{i}, \bar{Q}_{i}^{1 / 2}\right)$ is detectable $(i=$ $1,2, \ldots, N)$;

(II) A6 holds;

(III) $\left\|\bar{P}_{i}^{T} \bar{A}_{i j}\right\| \leq \beta_{i j}(i, j=1,2, \ldots, N, i \neq j)$;

(IV) matrix

$$
H=\left[\begin{array}{cccc}
\gamma_{1} & -2 \beta_{12} & \cdots & -2 \beta_{1 N} \\
-2 \beta_{21} & \gamma_{2} & \cdots & -2 \beta_{2 N} \\
\vdots & \vdots & \ddots & \vdots \\
-2 \beta_{N 1} & -2 \beta_{N 2} & \cdots & \gamma_{N}
\end{array}\right]
$$

is a nonsingular $M$ matrix, then the state vector $X_{i}(t)$ ( $i=1,2, \ldots, N)$ of System (35) (that is, the closed-loop system of (10)) is asymptotically stable to zero vector, where $\gamma_{i}=\lambda_{\min }\left(W_{i}\right)$, in which $W_{i}$ is any given positive definite matrix $(i=1,2, \ldots, N) . \bar{P}_{i}$ is the solution of the following equation:

$$
\left\{\begin{array}{l}
\bar{P}_{i}^{T}\left(\bar{A}_{i}-\bar{B}_{i} R_{i}^{-1} \widetilde{B}_{i 1}^{T} P_{i} U_{i}\right)+\left(\bar{A}_{i}-\bar{B}_{i} R_{i}^{-1} \widetilde{B}_{i 1}^{T} P_{i} U_{i}\right)^{T} \bar{P}_{i}=-W_{i}, \\
E_{i}^{T} \bar{P}_{i}=\bar{P}_{i}^{T} E_{i} \geq 0 .
\end{array}\right.
$$

Proof. Firstly, it is proved that the homogeneous system

$$
\begin{gathered}
\bar{E}_{i} \dot{X}_{i}(t)=\left(\bar{A}_{i}-\bar{B}_{i} R_{i}^{-1} \widetilde{B}_{i 1}^{T} P_{i} U_{i}\right) X_{i}(t)+\sum_{\substack{j=1 \\
j \neq i}}^{N} \bar{A}_{i j} X_{j}(t), \\
i=1,2, \ldots, N,
\end{gathered}
$$

corresponding to (35) is admissible.

In the light of Theorem $2,\left(\bar{E}_{i}, \bar{A}_{i}-\bar{B}_{i} R_{i}^{-1} \widetilde{B}_{i 1}^{T} P_{i} U_{i}\right)$ is admissible if (I) and (II) of this theorem hold. According to Lemma 3, given matrix $W_{i}>0$, there exists matrix $\bar{P}_{i}$ satisfying (38). Employing $\bar{E}_{i}$ and $\bar{P}_{i}$ to construct $V_{i}\left(\bar{E}_{i} X_{i}\right)=$ $X_{i}^{T} \bar{E}_{i}^{T} \bar{P}_{i} X_{i}$, it is clear that there is $V_{i}\left(\bar{E}_{i} X_{i}\right) \geq 0$. Differentiate $V_{i}\left(\bar{E}_{i} X_{i}\right)$ along System (39) trajectory to get 


$$
\begin{aligned}
\left.\dot{V}_{i}\right|_{(39)}= & \dot{X}_{i}^{T}(t) \bar{E}_{i}^{T} \bar{P}_{i} X_{i}(t)+X_{i}^{T}(t) \bar{E}_{i}^{T} \bar{P}_{i} \dot{X}_{i}(t) \\
= & \left(\bar{E}_{i} \dot{X}_{i}(t)\right)^{T} \bar{P}_{i} X_{i}(t)+X_{i}^{T}(t) \bar{P}_{i}^{T} \bar{E}_{i} \dot{X}_{i}(t) \\
= & X_{i}^{T}(t)\left[\left(\bar{A}_{i}-\bar{B}_{i} R_{i}^{-1} \widetilde{B}_{i 1}^{T} P_{i} U_{i}\right)^{T} \bar{P}_{i}\right. \\
& \left.+\bar{P}_{i}^{T}\left(\bar{A}_{i}-\bar{B}_{i} R_{i}^{-1} \widetilde{B}_{i 1}^{T} P_{i} U_{i}\right)\right] X_{i}(t) \\
& +2 X_{i}^{T} \bar{P}_{i}^{T} \sum_{j=1}^{N} \bar{A}_{i j} X_{j}(t) . \\
j \neq i &
\end{aligned}
$$

Note that $\gamma_{i}=\lambda_{\min }\left(W_{i}\right)=-\lambda_{\max }\left[\left(\bar{A}_{i}-\bar{B}_{i} R_{i}^{-1} \widetilde{B}_{i 1}^{T} P_{i} U_{i}\right)^{T}\right.$ $\left.\bar{P}_{i}+\bar{P}_{i}^{T}\left(\bar{A}_{i}-\bar{B}_{i} R_{i}^{-1} \widetilde{B}_{i 1}^{T} P_{i} U_{i}\right)\right]$ to obtain an estimate of $\left.\dot{V}_{i}\right|_{(39)}$ as follows:

$$
\left.\dot{V}_{i}\right|_{(39)} \leq-\gamma_{i} X_{i}^{T}(t) X_{i}(t)+2 X_{i}^{T} P_{i}^{T} \sum_{j=1}^{N} \bar{A}_{i j} X_{j}(t) .
$$

Continuously, utilizing the properties of norms and (III), we obtain

$$
\begin{gathered}
\left.\dot{V}_{i}\right|_{(39)} \leq-\gamma_{i}\left\|X_{i}(t)\right\|^{2}+2 \sum_{\substack{j=1 \\
j \neq i}}^{N}\left\|X_{i}^{T}(t)\right\|\left\|\bar{P}_{i}^{T} \bar{A}_{i j}\right\|\left\|X_{j}(t)\right\| \\
=-\left\|X_{i}(t)\right\|\left(\gamma_{i}\left\|X_{i}(t)\right\|-2 \sum_{\substack{j=1 \\
j \neq i}}^{N} \beta_{i j}\left\|X_{j}(t)\right\|\right) .
\end{gathered}
$$

Let $i=1,2, \ldots, N$ to get

$$
\left[\begin{array}{c}
\left.\dot{V}_{1}\right|_{(39)} \\
\left.\dot{V}_{2}\right|_{(39)} \\
\vdots \\
\left.\dot{V}_{N}\right|_{(39)}
\end{array}\right] \leq-\left[\begin{array}{cccc}
\left\|X_{1}(t)\right\| & 0 & \cdots & 0 \\
0 & \left\|X_{2}(t)\right\| & \cdots & 0 \\
\vdots & \vdots & \ddots & \vdots \\
0 & 0 & \cdots & \left\|X_{N}(t)\right\|
\end{array}\right] H\left[\begin{array}{c}
\left\|X_{1}(t)\right\| \\
\left\|X_{2}(t)\right\| \\
\vdots \\
\left\|X_{N}(t)\right\|
\end{array}\right] .
$$

Since (IV), that is, $H$ is a nonsingular $M$ matrix, there exists $K=\operatorname{diag}\left(k_{1}, k_{2}, \ldots, k_{N}\right)>0$ so that $K H+H^{T} K>0$ [28].

Using the diagonal elements of matrix $K$ and taking

$$
V=\sum_{i=1}^{N} k_{i} V_{i}=\left[\begin{array}{llll}
k_{1} & k_{2} & \cdots & k_{N}
\end{array}\right]\left[\begin{array}{c}
V_{1} \\
V_{2} \\
\vdots \\
V_{N}
\end{array}\right]
$$

as the Lyapunov function of System (39), the total derivative of $V$ along System (39) trajectory is

$$
\left.\dot{V}\right|_{(39)}=\left[\begin{array}{llll}
k_{1} & k_{2} & \cdots & k_{N}
\end{array}\right]\left[\begin{array}{c}
\left.\dot{V}_{1}\right|_{(39)} \\
\left.\dot{V}_{2}\right|_{(39)} \\
\vdots \\
\left.\dot{V}_{N}\right|_{(39)}
\end{array}\right] .
$$

Substitute (43) into (45) to obtain

$$
\begin{gathered}
\left.\dot{V}\right|_{(39) \leq-} \leq\left[\begin{array}{llll}
k_{1} & k_{2} & \cdots & k_{N}
\end{array}\right]\left[\begin{array}{cccc}
\left\|X_{1}(t)\right\| & 0 & \cdots & 0 \\
0 & \left\|X_{2}(t)\right\| & \cdots & 0 \\
\vdots & \vdots & \ddots & \vdots \\
0 & 0 & \cdots & \left\|X_{N}(t)\right\|
\end{array}\right] H\left[\begin{array}{c}
\left\|X_{1}(t)\right\| \\
\left\|X_{2}(t)\right\| \\
\vdots \\
\left\|X_{N}(t)\right\|
\end{array}\right] \\
=-\left[\left\|X_{1}(t)\right\|\left\|X_{1}(t)\right\| \cdots\left\|X_{1}(t)\right\|\right]\left(\frac{K H+H^{T} K}{2}\right)\left[\begin{array}{c}
\left\|X_{1}(t)\right\| \\
\left\|X_{2}(t)\right\| \\
\vdots \\
\left\|X_{N}(t)\right\|
\end{array}\right] .
\end{gathered}
$$


When $\lambda$ is utilized to represent the minimum eigenvalue of $\left(K H+H^{T} K\right) / 2$, there must be $\lambda>0$. Therefore, we obtain

$$
\left.\dot{V}\right|_{(39)} \leq-\lambda \sum_{i=1}^{N}\left\|X_{i}(t)\right\|^{2}=-\lambda\|X(t)\|^{2} .
$$

$X(t)=\left[\begin{array}{llll}X_{1}^{T}(t) & X_{2}^{T}(t) & \cdots & X_{N}^{T}(t)\end{array}\right]^{T}$ is the state vector of System (39). Therefore, the total derivative of $V$ along the trajectory of System (39) is negative definite. According to Lemma 3, System (39) is admissible.

Next, it is proven that $\lim _{t \rightarrow \infty} \eta_{i}(t)=0$ and $\eta_{i}(t)$ is bounded on $[0,+\infty)(i=1,2, \ldots, N)$. Note the Hypothesis A6, the desired tracking signal $y_{d}(t)$ is piecewise continuously differentiable, so $\dot{y}_{d}(t)$ has only the discontinuity of first kind at most. Naturally, $\dot{y}_{d}(t)$ is a bounded function. Besides, considering the hypothesis of $\lim _{t \longrightarrow \infty} \dot{y}_{d}(t)=0$, we only need to prove that $\lim _{t \longrightarrow \infty} g_{i}(t)=0$ and $g_{i}(t)$ is bounded.

Because $\widetilde{A}_{c i}$ is a stable matrix, there exist constants $\omega_{i}>0$ and $\alpha_{i}>0$, so that $\left\|\exp \left(\sigma \widetilde{A}_{c i}^{T}\right)\right\| \leq \omega_{i} \exp \left(-\alpha_{i} \sigma\right)$ holds for all $\sigma \geq 0$. Since $g_{i}(t)=R_{i}^{-1} \widetilde{B}_{i 1}^{T} \int_{0}^{l_{r}} \exp \left(\sigma \widetilde{A}_{c i}^{T}\right) P_{i} \widetilde{G}_{i 1} \dot{y}_{d}(t+\sigma) d \sigma$, there is

$$
\left\|g_{i}(t)\right\| \leq\left\|R_{i}^{-1} \widetilde{B}_{i 1}^{T}\right\| \int_{0}^{l_{r}}\left\|\exp \left(\sigma \widetilde{A}_{c i}^{T}\right)\right\|\left\|P_{i} \widetilde{G}_{i 1}\right\|\left\|\dot{y}_{d}(t+\sigma)\right\| \mathrm{d} \sigma .
$$

Substitute $\left\|\exp \left(\sigma \widetilde{A}_{c i}^{T}\right)\right\| \leq \omega_{i} \exp \left(-\alpha_{i} \sigma\right)$ to get

$$
\begin{aligned}
\left\|g_{i}(t)\right\| & \leq\left\|R_{i}^{-1} \widetilde{B}_{i 1}^{T}\right\| \int_{0}^{l_{r}} \omega_{i} \exp \left(-\alpha_{i} \sigma\right)\left\|P_{i} \widetilde{G}_{i 1}\right\|\left\|\dot{y}_{d}(t+\sigma)\right\| \mathrm{d} \sigma \\
& \leq \omega_{i}\left\|R_{i}^{-1} \widetilde{B}_{i 1}^{T}\right\|\left\|P_{i} \widetilde{G}_{i 1}\right\| \int_{0}^{l_{r}}\left\|\dot{y}_{d}(t+\sigma)\right\| \mathrm{d} \sigma
\end{aligned}
$$

For any given $\varepsilon>0$, due to $\lim _{t \longrightarrow \infty} \dot{y}_{d}(t)=0$, there exists $T_{0}>0$ such that when $t \geq T_{0}$, there is $\left\|\dot{y}_{d}(t)\right\|<$ $\left(1 /\left(\left(1+\omega_{i}\left\|R_{i}^{-1} \widetilde{B}_{i 1}^{T}\right\|\left\|P_{i} \widetilde{G}_{i 1}\right\|\right)\left(1+l_{r}\right)\right)\right) \varepsilon$, so when $t \geq T_{0}$, there is

$$
\begin{aligned}
\left\|g_{i}(t)\right\| & <\omega_{i}\left\|R_{i}^{-1} \widetilde{B}_{i 1}^{T}\right\|\left\|P_{i} \widetilde{G}_{i 1}\right\| \int_{0}^{l_{r}} \frac{1}{\left(1+\omega_{i}\left\|R_{i}^{-1} \widetilde{B}_{i 1}^{T}\right\|\left\|P_{i} \widetilde{G}_{i 1}\right\|\right)\left(1+l_{r}\right)} \varepsilon \mathrm{d} \sigma \\
& =\frac{\left(\omega_{i}\left\|R_{i}^{-1} \widetilde{B}_{i 1}^{T}\right\|\left\|P_{i} \widetilde{G}_{i 1}\right\|\right) l_{r}}{\left(1+\omega_{i}\left\|R_{i}^{-1} \widetilde{B}_{i 1}^{T}\right\|\left\|P_{i} \widetilde{G}_{i 1}\right\|\right)\left(1+l_{r}\right)} \varepsilon<\varepsilon .
\end{aligned}
$$

This proves $\lim _{t \longrightarrow \infty} g_{i}(t)=0$.

Secondly, $\exp \left(\sigma \widetilde{A}_{c i}^{T}\right) P_{i} \widetilde{G}_{i 1} \dot{y}_{d}(t+\sigma)$ has only the discontinuity of the first kind at most on the basis of the property of $\dot{y}_{d}(t)$, so $g_{i}(t)$ is a continuous function [29]. As a result, $g_{i}(t)$ is bounded on $[0,+\infty)$ since $g_{i}(t)$ is continuous and $\lim _{t \rightarrow \infty} g_{i}(t)=0$ [30]. This proves that $\lim _{t \rightarrow \infty} \eta_{i}(t)=$ 0 and $\eta_{i}(t)$ is bounded on $[0,+\infty)$.

Because System (39) is admissible, $\lim _{t \longrightarrow \infty} \eta_{i}(t)=0$ and $\eta_{i}(t)$ is bounded on $[0,+\infty)$, according to [31], there is $\lim _{t \rightarrow \infty} X_{i}(t)=0(i=1,2, \ldots, N)$ in System (35). Hence, Theorem 3 is proved.
Below, we adopt the relevant parameters of singular interconnected System (1) to give the conditions, which ensure that $\left(\bar{E}_{i}, \bar{A}_{i}, \bar{B}_{i}\right)$ is stabilizable and $\left(\bar{E}_{i}, \bar{A}_{i}, \bar{Q}_{i}{ }^{1 / 2}\right)$ is detectable.

Lemma 4 (see [32]). The sufficient and necessary condition for $\left(\bar{E}_{i}, \bar{A}_{i}, \bar{B}_{i}\right)$ to be stabilizable is that rank $\left[\begin{array}{cc}A_{i} & B_{i} \\ C_{i} & 0\end{array}\right]=n_{i}+$ $p$ and $\left(E_{i}, A_{i}, B_{i}\right)$ is stabilizable $(i=1,2, \ldots, N)$.

Lemma 5 (see [32]). The sufficient and necessary condition for $\left(\bar{E}_{i}, \bar{A}_{i}, \bar{Q}_{1}^{1 / 2}\right)$ to be detectable is that $\left(E_{i}, A_{i}, C_{i}\right)$ is detectable $(i=1,2, \ldots, N)$.

Lemmas 4 and 5 can be proved by a method similar to that in reference [32], so the proof is omitted here.

To sum up, one of the main theorems in this paper is as follows.

Theorem 4. Suppose

(I) A1-A6 hold

(II) $\left\|\bar{P}_{i}^{T} \bar{A}_{i j}\right\| \leq \beta_{i j}(i, j=1,2, \ldots, N, i \neq j)$

(III) $H$ is a nonsingular $M$ matrix

(IV) $Q_{e_{i}}>0, R_{i}>0(i=1,2, \ldots, N)$

(V) Let $y_{d}(k)=0, x_{i}(t)=0, u_{i}(t)=0(i=1,2, \ldots, N)$ for $t<0$

Then, the controller with the preview effect, which enables the output signal of (1) to track the desired tracking signal asymptotically, is given as

$$
u(t)=\left[\begin{array}{llll}
u_{1}^{T}(t) & u_{2}^{T}(t) & \cdots & u_{N}^{T}(t)
\end{array}\right]^{T},
$$

where

$$
\begin{gathered}
u_{i}(t)=u_{i}(0)-F_{e_{i}} \int_{0}^{t} e_{i}(\sigma) d \sigma-F_{x_{i}}\left[x_{i}(t)-x_{i}(0)\right]+\bar{g}_{i}(t), \\
i=1,2, \ldots, N
\end{gathered}
$$

in which $F_{e_{i}}=R_{i}^{-1} \widetilde{B}_{i 1}^{T} P_{i} U_{e i}, \quad F_{x_{i}}=R_{i}^{-1} \widetilde{B}_{i 1}^{T} P_{i} U_{x i}$, and $U_{i}=$ $\left[\begin{array}{ll}U_{e i} & U_{x i}\end{array}\right]$; the expression of $\bar{g}_{i}(t) \in R^{p}$ is $\bar{g}_{i}(t)=$ $R_{i}^{-1} \widetilde{B}_{i 1}^{T} \int_{0}^{l_{r}} \exp \left(\sigma \widetilde{A}_{c i}^{T}\right) P_{i} \widetilde{G}_{i 1}\left[y_{d}(t+\sigma)-y_{d}(\sigma)\right] d \sigma$.

Proof. According to Lemmas 4 and 5, when (I)-(IV) is true here, all conditions of Theorems 1 to 3 are satisfied, so the conclusion is valid. Thus, (32) gives the controller of the error system, in which the component $\dot{u}_{i}(i=1,2, \ldots, N)$ is determined by (28) of Theorem 2 . We now derive the controller of the original system from (32) and (28).

Note that (28) is

$$
\begin{aligned}
\dot{u}_{i}(t)= & -F_{e_{i}} e_{i}(t)-F_{x_{i}} \dot{x}_{i}(t) \\
& +R_{i}^{-1} \widetilde{B}_{i 1}^{T} \int_{0}^{l_{r}} \exp \left(\sigma \widetilde{A}_{c i}^{T}\right) P_{i} \widetilde{G}_{i 1} \dot{y}_{d}(t+\sigma) \mathrm{d} \sigma .
\end{aligned}
$$

Integrate on $[0, t]$ to get 


$$
\begin{aligned}
u_{i}(t)-u_{i}(0)= & -F_{e_{i}} \int_{0}^{t} e_{i}(\sigma) \mathrm{d} \sigma-F_{x_{i}}\left[x_{i}(t)-x_{i}(0)\right] \\
& +R_{i}^{-1} \widetilde{B}_{i 1}^{T} \int_{0}^{t}\left[\int_{0}^{l_{r}} \exp \left(\sigma \widetilde{A}_{c i}^{T}\right) P_{i} \widetilde{G}_{i 1} \dot{y}_{d}(t+\sigma) \mathrm{d} \sigma\right] \mathrm{d} t .
\end{aligned}
$$

By exchanging the integration order of the last item on the right side of the upper formula and then integrating it, we can get (52). Then, combining $u_{i}(t)(i=1,2, \ldots, N)$, the controller of System (1) is attained, that is, (51).

Remark 4. In (52), $F_{x_{i}} x_{i}(t)$ is the state feedback, $F_{e_{i}} \int_{0}^{t} e(\sigma) \mathrm{d} \sigma$ is the integrator, and $\bar{g}_{i}(t)$ is the preview feedforward of reference information; moreover, $u_{i}(0)$ is the initial value of input and $F_{x_{i}} x_{i}(0)$ is the compensation of initial value.

\section{Numerical Simulation}

5.1. Numerical Simulation Method. In this section, the numerical simulation of a singular system

$$
\left\{\begin{array}{l}
E \dot{x}(t)=A x(t)+B u(t) \\
y(t)=C x(t)
\end{array}\right.
$$

is discussed. Here, matrices $A, B$, and $C$ have appropriate dimensions, $A$ is a square matrix, and $E$ is a singular matrix and satisfies $\operatorname{rank}(E)=q<n$. Let the state feedback be $u(t)=F x(t)$.

Because (55) contains the singular matrix $E$, it is impossible to calculate the state value of the next moment from System (55) and $u(t)=F x(t)$ by the usual direct discretization method, as normal systems do. Therefore, an algorithm is proposed to solve this problem.

Taking the sampling interval as $h$, it is noted that

$$
\dot{x}((k+1) h)=\frac{x(k h)-x((k+1) h)}{-h} .
$$

At $t=(k+1) h$, equation (55) is discretized to obtain

$$
E \frac{x(k h)-x((k+1) h)}{-h}=A x((k+1) h)+B u((k+1) h),
$$

that is,

$$
(E-A h) x((k+1) h)=E x(k h)+B h u((k+1) h) .
$$

For equation (58), an appropriate sampling interval $h$ can be chosen so that matrix $(E-A h)$ is nonsingular. Then, (58) can be written as

$$
x((k+1) h)=(E-A h)^{-1}[E x(k h)+B h u((k+1) h)] .
$$

Unfortunately, since the right side of (59) contains the term $u((k+1) h)$, besides, $u(t)=F x(t)$, it is known that $u((k+1) h)$ is related to $x((k+1) h)$; therefore, equation $(59)$ cannot be calculated. To overcome this difficulty, we take $u(k h)$ as the approximate value of $u((k+1) h)$ and then substitute it into (59) to obtain the following iteration scheme:

$$
x((k+1) h)=(E-A h)^{-1}[E x(k h)+B h u(k h)] .
$$

The rationality of replacing $u((k+1) h)$ with $u(k h)$ is explained below. Notice that if the output of the closed-loop system can track the reference signal, there are $x(\infty)$ and $u(\infty)$ such that

$$
\left\{\begin{array}{l}
0=A x(\infty)+B u(\infty) \\
y(\infty)=C x(\infty)
\end{array}\right.
$$

By adopting (56), (60) can be written as $E \dot{x}((k+1) h)=A x((k+1) h)+B u(k h)$. Letting $k \longrightarrow \infty$, we get the same relation. Therefore, this method is reasonable. In other words, when $k$ is large, there is $u((k+1) h) \approx u(k h)$.

Notice that the output equation is $y(t)=C x(t)$, and then the iterative scheme of (55) is

$$
\left\{\begin{array}{l}
x((k+1) h)=(E-A h)^{-1}[E x(k h)+B h u(k h)], \\
y(k h)=C x(k h) .
\end{array}\right.
$$

The convergence condition of the iterative scheme (62) is given below. The state feedback $u(k h)=F x(k h)$ is substituted into the equation of state to obtain the following closed-loop system:

$$
x((k+1) h)=(E-A h)^{-1}(E-h B F) x(k h) .
$$

Obviously, a sufficient condition for the convergence of the iteration scheme (62) is that the spectral radius of $(E-A h)^{-1}(E-h B F)$ (i.e., the maximum value of the absolute value of the eigenvalue) is less than 1 [33].

5.2. Simulation Example. In this section, the effectiveness of the designed controller is verified by numerical simulation.

Considering the singular interconnected system with two subsystems (i.e., $N=2$ ) $n_{1}=3$ and $n_{2}=2$, the coefficient matrices are

$$
\begin{aligned}
E_{1} & =\left[\begin{array}{lll}
1 & 0 & 0 \\
0 & 0 & 0 \\
0 & 0 & 1
\end{array}\right], \\
A_{1} & =\left[\begin{array}{ccc}
-1 & 1 & 0 \\
-2 & -4 & 0 \\
1.5 & 0 & -1
\end{array}\right], \\
A_{12} & =\left[\begin{array}{cc}
0.4 & 0.1 \\
0.5 & 5 \\
-0.6 & 0.1
\end{array}\right], \\
B_{1} & =\left[\begin{array}{l}
1 \\
2 \\
1
\end{array}\right],
\end{aligned}
$$




$$
\begin{aligned}
& C_{1}=\left[\begin{array}{lll}
1 & 1 & 1.5
\end{array}\right], \\
& E_{2}=\left[\begin{array}{ll}
0 & 0 \\
0 & 1
\end{array}\right] \text {, } \\
& A_{2}=\left[\begin{array}{cc}
-2 & 0 \\
1 & -1
\end{array}\right] \text {, } \\
& A_{21}=\left[\begin{array}{ccc}
0.1 & 0.2 & -0.4 \\
0.1 & 0.5 & 1
\end{array}\right] \text {, } \\
& B_{1}=\left[\begin{array}{l}
1 \\
2
\end{array}\right] \text {, } \\
& C_{2}=\left[\begin{array}{ll}
1 & 1.5
\end{array}\right] \text {. }
\end{aligned}
$$

(7) be

Let the weight matrix of the performance index function

$$
\begin{aligned}
Q_{e_{1}} & =50, \\
R_{1} & =10, \\
Q_{e_{2}} & =50, \\
R_{2} & =10 .
\end{aligned}
$$

$y_{d}(t)$ can be chosen as

$$
y_{d}(t)= \begin{cases}0, & t>2.5, \\ 2(t-2), & 2 \leq t<2.5 \\ 1, & t>2.5 .\end{cases}
$$

Take the initial state be $x_{1}(0)=\left[\begin{array}{lll}0.02 & 0 & 0\end{array}\right]^{T}, u_{1}(0)=0$, $x_{1}(0)=\left[\begin{array}{ll}0 & 0.01\end{array}\right]^{T}$, and $u_{2}(0)=0$. Select $\alpha_{1}=0.3$ and $\alpha_{2}=0.7$. It is easy to verify that the given system and the reference signal meet A1-A6.

We conducted numerical simulation for $l_{r}=0$ (without reference signal preview), $l_{r}=0.5$, and $l_{r}=1.2$, respectively. The solution of the Riccati equation for two isolated subsystems and the feedback gain matrix of the controller is obtained by using MATLAB as follows:

$$
\begin{aligned}
P_{1} & =\left[\begin{array}{lll}
21.217420616941663 & 6.816120898775491 & 3.290565711834407 \\
6.816120898775491 & 3.969175332838401 & 2.538387150184135 \\
3.290565711834407 & 2.538387150184135 & 1.950469194330368
\end{array}\right], \\
P_{2} & =\left[\begin{array}{ll}
18.374963043673894 & 5.269279301264373 \\
5.269279301264373 & 2.934066776906499
\end{array}\right], \\
F_{e_{1}} & =2.236067977499793, \\
F_{x_{1}} & =\left[\begin{array}{lll}
0.710937379759689 & 0 & 1.118481650750235
\end{array}\right], \\
F_{e_{2}} & =2.236067977499788, \\
F_{x_{2}} & =\left[\begin{array}{lll}
0 & 0.996980659289843
\end{array}\right] .
\end{aligned}
$$

Let us take

$$
\begin{aligned}
& W_{1}=\left[\begin{array}{ccc}
10 & 0 & 0 \\
0 & 10 & 0 \\
0 & 0 & 10
\end{array}\right], \\
& W_{2}=\left[\begin{array}{cccc}
10 & 0 & 0 & 0 \\
0 & 10 & 0 & 0 \\
0 & 0 & 10 & 0 \\
0 & 0 & 0 & 10
\end{array}\right] .
\end{aligned}
$$

Obviously, there are $\gamma_{1}=\lambda_{\min }\left(W_{1}\right)=10$ and $\gamma_{2}=\lambda_{\min }\left(W_{2}\right)=10$. The solution of the singular Lyapunov equation is obtained by using MATLAB:

$$
\begin{aligned}
\bar{P}_{1} & =\left[\begin{array}{cccc}
7.647751127599625 & -0.177729150439247 & 0 & 1.473871111233592 \\
-0.177729150439237 & 3.477348552026456 & 0 & 0.368592368778278 \\
0.469963008352726 & -0.244431011953004 & 1.250000000000000 & -0.238435161715929 \\
1.473871111233594 & 0.368592368778280 & 0 & 3.460928523839078
\end{array}\right], \\
\bar{P}_{2}= & {\left[\begin{array}{ccc}
7.540791381135387 & 0 & 0.504302903522732 \\
1.227462170454325 & 2.500000000000000 & -0.028058524738017 \\
0.504302903522733 & 0 & 1.932031695225841
\end{array}\right] . }
\end{aligned}
$$




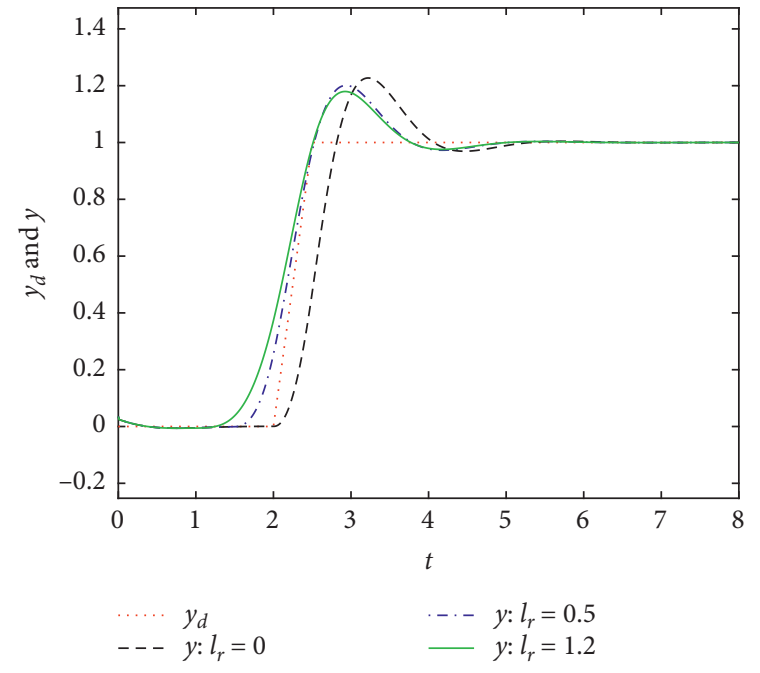

FIGURE 1: The output response of singular interconnected systems.

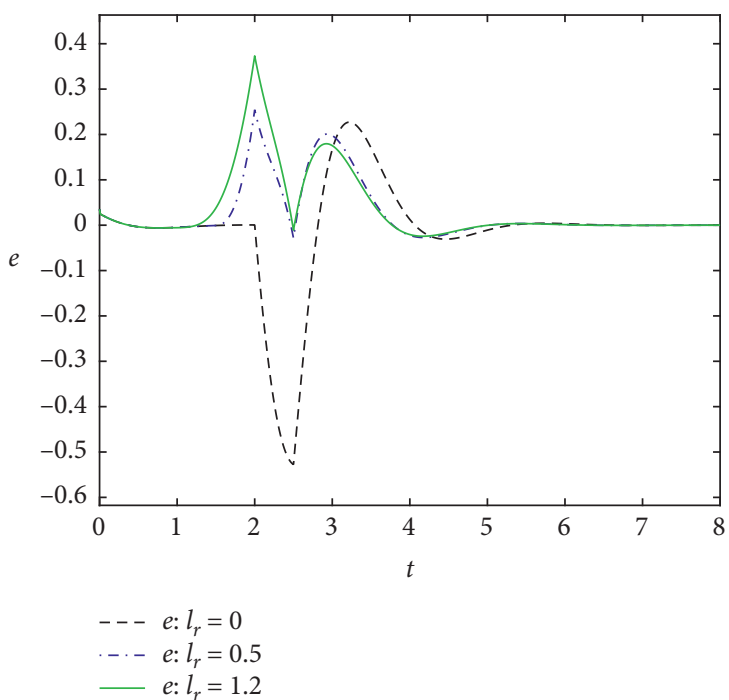

FIGURE 2: The tracking errors of singular interconnected systems.

Let $\beta_{i j}=\left\|\bar{P}_{i}^{T} \bar{A}_{i j}\right\|_{2}$, then $\beta_{12}=6.839132471138595$ and $\beta_{12}=2.685100253336826$. So,

$$
H=\left[\begin{array}{cc}
10.000000000000000 & -13.678264942277190 \\
-5.370200506673653 & 10.000000000000000
\end{array}\right] \text {. }
$$

The eigenvalues of $H$ are 18.570590721965054 and 1.429409278034949 , which means that $H$ is a nonsingular $M$ matrix. In conclusion, the conditions of Theorem 4 are all satisfied.

Selecting the sampling interval $h=0.01$, the calculation shows that $E-A h$ is nonsingular and the spectral radius of $(E-A h)^{-1}\left(E-h B F_{x}\right)$ is less than 1 . The tracking effect of System (1) is shown in Figure 1.

As can be seen from Figure 1, with the gradual increase in time $t$, the output signals of the singular

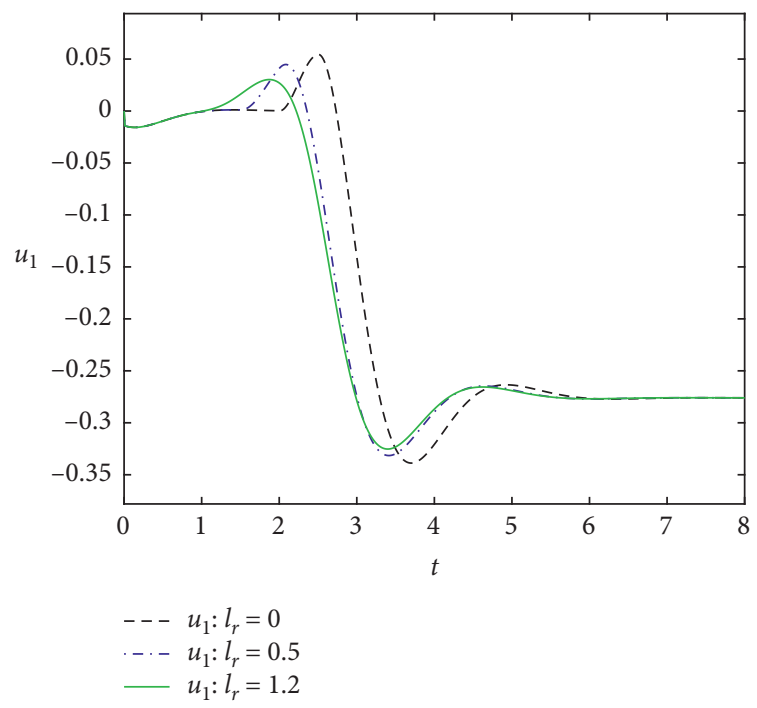

Figure 3: Control input components $u_{1}(t)$ of singular interconnected systems.

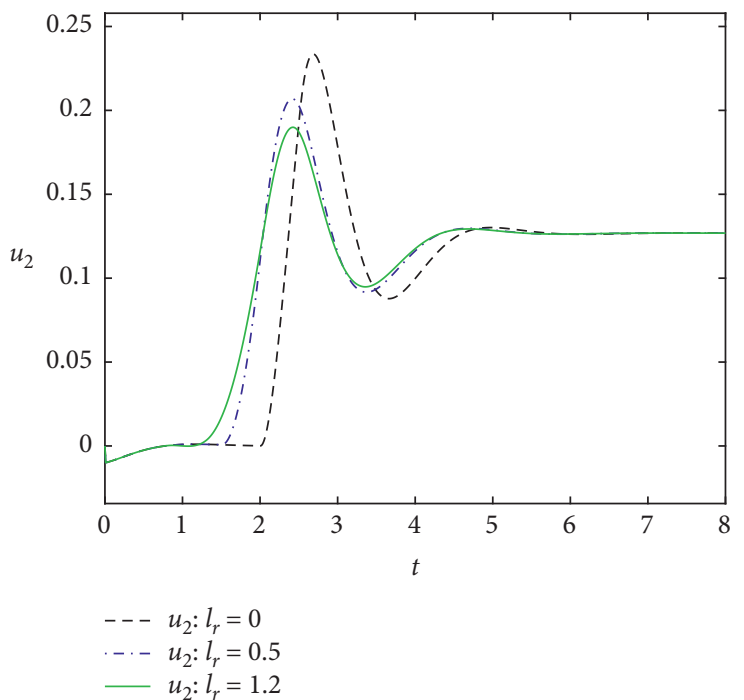

Figure 4: Control input components $u_{2}(t)$ of singular interconnected systems.

interconnected system (1) can asymptotically track the desired tracking signal under different preview lengths. In addition, with the increase in the preview length, overshoot and adjustment time are decreasing. The tracking error of singular interconnected systems is given in Figure 2. It can be found from Figure 2 that, compared with the controller with no preview effect (i.e., $l_{r}=0$ ), the controller with the preview effect can reduce the overall tracking error.

Figures 3 and 4 depict the control input component diagram of the singular interconnected System (1). It can be seen from the graph that the increase in the preview length will not make the control input change dramatically but makes the control input change smoothly. 


\section{Conclusion}

In this paper, the basic theory of preview control is extended to continuous-time singular interconnected systems and the problem of preview tracking control for such systems is studied. With the help of decomposition theory of largescale systems, several isolated subsystems are obtained by deleting related terms. Furthermore, the common methods of preview control theory are adopted to construct error systems for isolated subsystems, and the tracking problem is transformed into a regulation problem. For each isolated error system, the preview controller is designed and the obtained controllers are combined as the controllers of the singular interconnected system error system. By constructing Lyapunov functions and using the properties of nonsingular M-matrices, the stability of closed-loop error interconnected systems is discussed, and the criterion theorem to ensure its stability is given. Finally, the sufficient conditions for the existence of preview controllers and controllers in the original singular interconnected system are derived.

Furthermore, a numerical simulation algorithm for continuous-time singular systems is proposed, which does not depend on the restricted equivalent transformation and is suitable for the numerical simulation of all continuoustime singular systems. The theoretical results and numerical simulation show that the designed controller is able to make the output of the system to track the desired tracking signal without a static error, and the tracking performance is improved with the increase in the preview length.

\section{Data Availability}

The data used to support the findings of this study are included within the article.

\section{Conflicts of Interest}

The authors declare that they have no conflicts of interest.

\section{Acknowledgments}

This work was supported by the Oriented Award Foundation for Science and Technological Innovation, Inner Mongolia Autonomous Region, China (Grant no. 2012), and National Key R\&D Program of China (Grant no. 2017YFF0207401).

\section{References}

[1] F. L. Lewis, "A survey of linear singular systems," Circuits, Systems, and Signal Processing, vol. 5, no. 1, pp. 3-36, 1986.

[2] L. Dai, Singular Control Systems, Springer-Verlag, Berlin, Germany, 1989.

[3] C. Liu, Q. Zhang, Y. Zhang, and X. Duan, "Bifurcation and control in a differential-algebraic harvested prey-predator model with stage structure for predator," International Journal of Bifurcation and Chaos, vol. 18, no. 10, pp. 31593168, 2008.

[4] D. Cobb, "Controllability, observability, and duality in singular systems," IEEE Transactions on Automatic Control, vol. 29, no. 12, pp. 1076-1082, 1984.
[5] A. Ailon, "On the reduced-order causal observer for generalized control systems," International Journal of Control, vol. 57, no. 6, pp. 1311-1323, 1993.

[6] J. Y. Ishihara and M. H. Terra, "On the Lyapunov theorem for singular systems," IEEE Transactions on Automatic Control, vol. 47, no. 11, pp. 1926-1930, 2002.

[7] N. Kumaresan and P. Balasubramaniam, "Optimal control for stochastic nonlinear singular system using neural networks," Computers \& Mathematics with Applications, vol. 56, no. 9, pp. 2145-2154, 2008.

[8] M. Fang, "Delay-dependent stability analysis for discrete singular systems with time-varying delays," Acta Automatica Sinica, vol. 36, no. 5, pp. 751-755, 2010.

[9] I. Masubuchi, Y. Kamitane, A. Ohara, and N. Suda, " $H_{\infty}$ control for descriptor systems: a matrix inequalities approach," Automatica, vol. 33, no. 4, pp. 669-673, 1997.

[10] C. Chen and Y. Liu, "Lyapunov stability analysis of linear singular dynamical systems," in Proceedings of the IEEE International Conference on Intelligent Processing Systems, IEEE, Beijing, China, October 1997.

[11] S. Wo, Y. Zou, M. Sheng, and S. Xu, "Robust control for discrete-time singular large-scale systems with parameter uncertainty," Journal of the Franklin Institute, vol. 344, no. 2, pp. 97-106, 2007.

[12] R. Tang, X. Lü, Y. Zhai, and C. Gong, "Optimal tracking control for nonlinear large-scale systems with persistent disturbances," Journal of Control Theory and Applications, vol. 8, no. 4, pp. 515-520, 2010.

[13] D. D. Siljak, Large-Scale Dynamic Systems Stability and Structure, Dover Publications, New York, NY, USA, 2007.

[14] Q. Zhang, Decentralized Control and Robust Control of Generalized Large-Scale Systems, Northwestern Polytechnical University Press, Xian, China, 1997.

[15] J. H. Park and S. G. Lee, "Robust decentralized stabilization of uncertain large-scale discrete-time systems," International Journal of Systems Science, vol. 33, no. 8, pp. 649-654, 2002.

[16] G. Lu and D. W. C. Ho, "Continuous stabilization controllers for singular bilinear systems: the state feedback case," Automatica, vol. 42, no. 2, pp. 309-314, 2006.

[17] C. Chen and J. Ding, "The stabilization of singular linear largescale control systems with output feedbacks," Advanced Materials Research, vol. 383-390, pp. 72-78, 2012.

[18] T. Katayama and T. Hirono, "Design of an optimal servomechanism with preview action and its dual problem," International Journal of Control, vol. 45, no. 2, pp. 407-420, 1987.

[19] F. Liao, Y. Y. Tang, H. Liu, and Y. Wang, "Design of an optimal preview controller for continuous-time systems," International Journal of Wavelets, Multiresolution and Information Processing, vol. 9, no. 4, pp. 655-673, 2011.

[20] J. Wu, F. Liao, and M. Tomizuka, "Optimal preview control for a linear continuous-time stochastic control system in finite-time horizon," International Journal of Systems Science, vol. 48, no. 1, pp. 129-137, 2017.

[21] L. Li and F. Liao, "Parameter-dependent preview control with robust tracking performance," IET Control Theory \& Applications, vol. 11, no. 1, pp. 38-46, 2016.

[22] N. Birla and A. Swarup, "Optimal preview control: a review," Optimal Control Applications and Methods, vol. 36, no. 2, pp. 241-268, 2015.

[23] P. Li, J. Lam, and K. C. Cheung, "Multi-objective control for active vehicle suspension with wheelbase preview," Journal of Sound and Vibration, vol. 333, no. 21, pp. 5269-5282, 2014. 
[24] S. Yim, "Design of preview controllers for active roll stabilization," Journal of Mechanical Science and Technology, vol. 32, no. 4, pp. 1805-1813, 2018.

[25] R. Takase, Y. Hamada, and T. Shimomura, "Aircraft gust alleviation preview control with a discrete-time LPV model," SICE Journal of Control, Measurement, and System Integration, vol. 11, no. 3, pp. 190-197, 2018.

[26] G. Duan, Analysis and Design of Descriptor Linear Systems, Springer-Verlag, New York, NY, USA, 2010.

[27] D. L. J. Debeljkovic and I. M. Buzurovic, "Lyapunov stability of linear continuous singular systems: an overview," International Journal of Information and Systems Sciences, vol. 7, no. $2-3$, pp. $247-268,2011$.

[28] R. J. Plemmons, " $M$-matrix characterizations $I$-nonsingular M-matrices," Linear Algebra and Its Applications, vol. 18, no. 12, pp. 175-188, 1977.

[29] K. L. Chung, A Course in Probability Theory, Academic Press, London, UK, 2001.

[30] V. A. Zorich, Mathematical Analysis I, Springer-Verlag, New York, NY, USA, 2004.

[31] Y. Lu, F. Liao, J. Deng, and C. Pattinson, "Cooperative optimal preview tracking for linear descriptor multi-agent systems," Journal of the Franklin Institute, vol. 356, no. 2, pp. 908-934, 2018.

[32] F. Liao, Z. Ren, M. Tomizuka, and J. Wu, "Preview control for impulse-free continuous-time descriptor systems," International Journal of Control, vol. 88, no. 6, pp. 1142-1149, 2015.

[33] D. Faires and R. L. Burden, Numerical Methods, Brooks Cole Publishing Co., Pacific Grove, CA, USA, 2nd edition, 2002. 


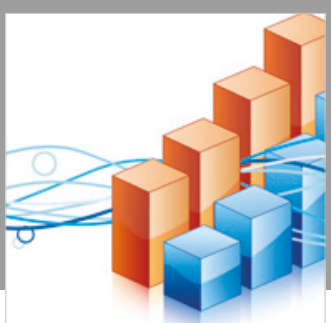

Advances in

Operations Research

\section{-n-m}
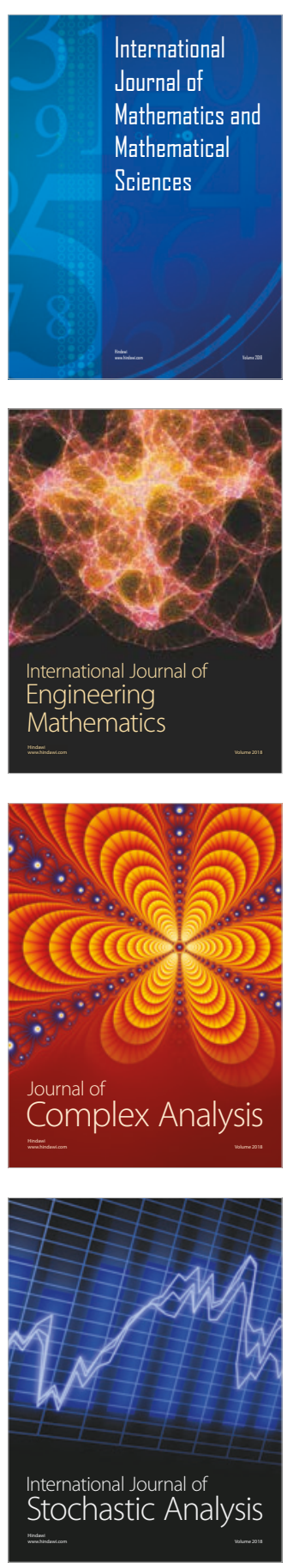
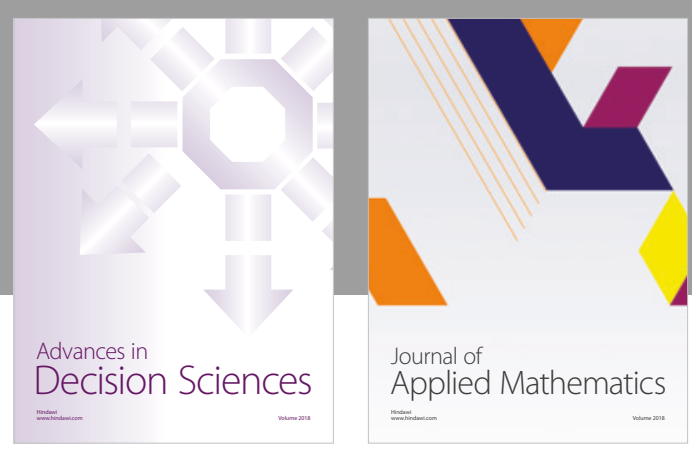

Journal of

Applied Mathematics
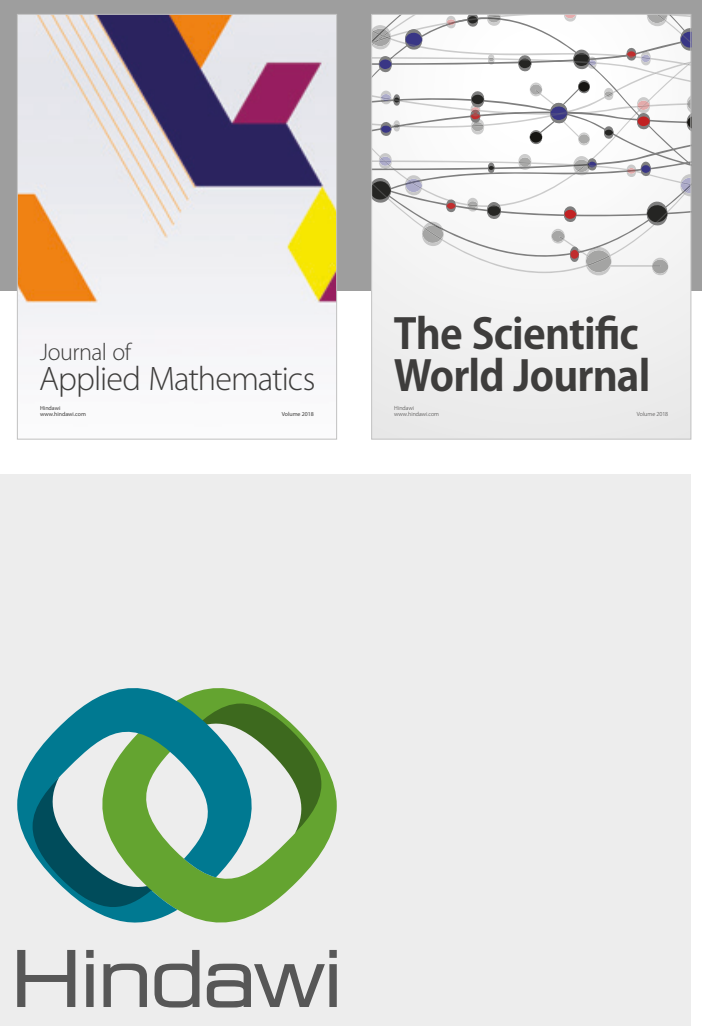

Submit your manuscripts at

www.hindawi.com

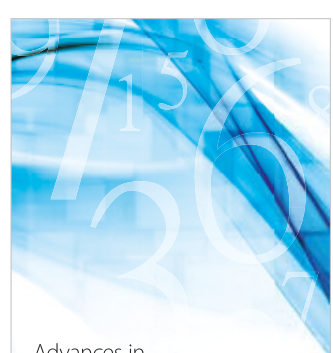

Advances in
Numerical Analysis
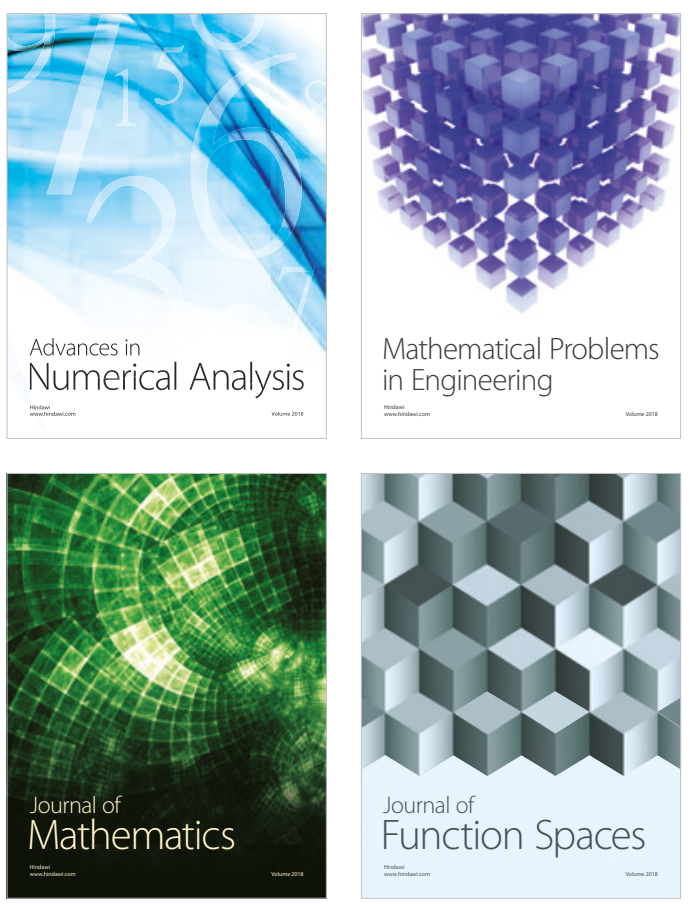

Mathematical Problems in Engineering

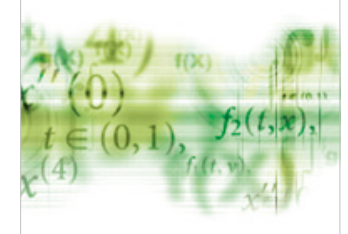

International Journal of

Differential Equations

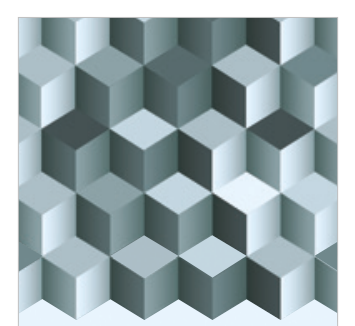

Journal of

Function Spaces

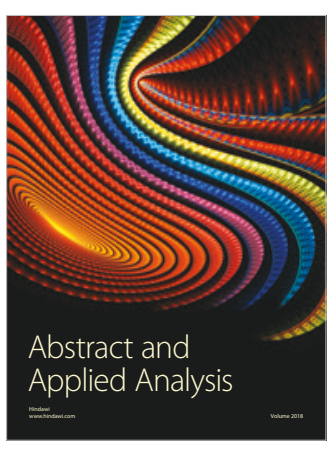

The Scientific

World Journal

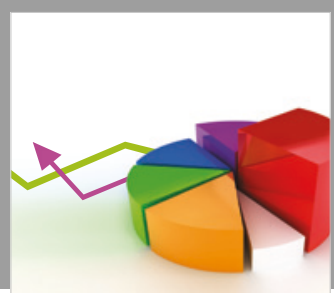

Journal of

Probability and Statistics
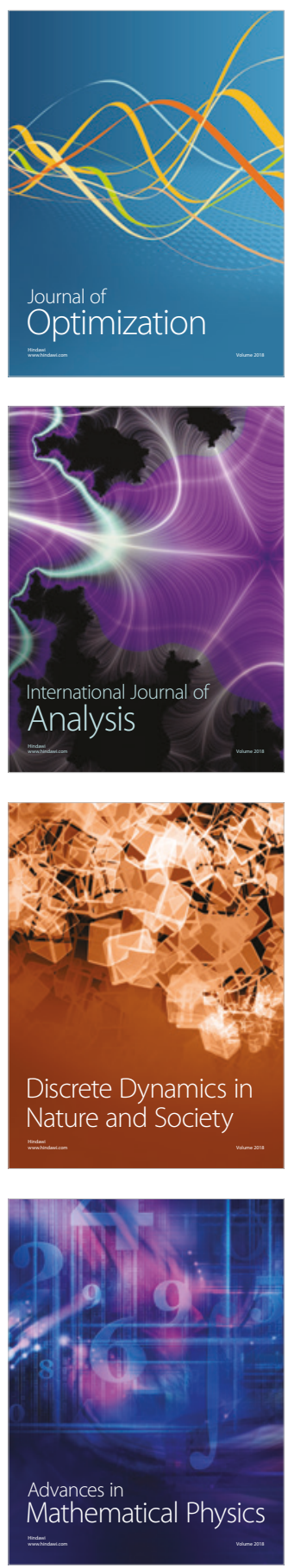